Editorial

\title{
Optimal Control: Theory and Application to Science, Engineering, and Social Sciences
}

\author{
Davide La Torre, ${ }^{1,2}$ Herb Kunze, ${ }^{3}$ Manuel Ruiz-Galan, ${ }^{4}$ \\ Tufail Malik, ${ }^{1}$ and Simone Marsiglio ${ }^{5}$ \\ ${ }^{1}$ Department of Applied Mathematics and Sciences, Khalifa University, Abu Dhabi 127788, UAE \\ ${ }^{2}$ Department of Economics, Management, and Quantitative Methods, University of Milan, 20122 Milan, Italy \\ ${ }^{3}$ Department of Mathematics and Statistics, University of Guelph, Guelph, ON, Canada N1G 2W1 \\ ${ }^{4}$ Department of Applied Mathematics, University of Granada, 18071 Granada, Spain \\ ${ }^{5}$ School of Accounting, Economics and Finance, University of Wollongong, Wollongong, NSW 2522, Australia
}

Correspondence should be addressed to Davide La Torre; davide.latorre@kustar.ac.ae

Received 12 March 2015; Accepted 12 March 2015

Copyright (C) 2015 Davide La Torre et al. This is an open access article distributed under the Creative Commons Attribution License, which permits unrestricted use, distribution, and reproduction in any medium, provided the original work is properly cited.

An optimal control problem entails the identification of a feasible scheme, policy, program, strategy, or campaign, in order to achieve the optimal possible outcome of a system. More formally, an optimal control problem means endogenously controlling a parameter in a mathematical model to produce an optimal output, using some optimization technique. The problem comprises an objective (or cost) functional, which is a function of the state and control variables, and a set of constraints. The problem seeks to optimize the objective function subject to the constraints construed by the model describing the evolution of the underlying system. The two popular solution techniques of an optimal control problem are Pontryagin's maximum principle and the HamiltonJacobi-Bellman equation [1].

Optimal control has become a highly established research front in recent years with numerous contributions to the theory, in both deterministic and stochastic contexts. Its application to diverse fields such as biology, economics, ecology, engineering, finance, management, and medicine cannot be overlooked (see, e.g., [1-12]). The associated mathematical models are formulated, for example, as systems of ordinary, partial, or stochastic differential equations or discrete dynamical systems, for both scalar and multicriteria decision-making contexts.

This special issue is aimed at creating a multidisciplinary forum of discussion on recent advances in theory as well as applications. In response to the call for papers, 58 papers were received. After a rigorous refereeing process, 7 papers were accepted for publication in this special issue. The articles included in the issue cover novel contributions to optimal control theory as well as a broad spectrum of applications of optimal control from finance to resource management to engineering to marketing.

The paper by Z. Lu and X. Huang investigates discretization of general linear hyperbolic optimal control problems and derives a priori error estimates for mixed finite element approximation of such optimal control problems.

The paper by R. Wu et al. proposes, analyzes, and solves an optimal control exploiting model of renewable resources based on effective utilization rate and illustrates its solution numerically.

The paper by N. Zulkarnain et al. compares the performance of an active antiroll bar system in an automobile using two control models, namely, the linear quadratic Gaussian and the composite nonlinear feedback controller.

There are three works focusing on applications in economics and finance. The paper by J. Ma et al. studies the dynamics of an oligopoly model considering the output of an upstream monopoly and prices of two downstream oligopolies; it is shown that the long-run average profits of the three firms are optimal in the region of stability of the Nash equilibrium point. The paper by D.-L. Sheng et al. uses the 
constant elasticity of variance model to describe the dynamics of the risk asset and establish a Hamilton-Jacobi-Bellman equation to solve the associated optimal control problem. An asset and liability management problem with stochastic interest rate is examined in the paper by $\mathrm{H}$. Chang et al., who investigate an optimal investment strategy to maximize the expected utility of the terminal surplus.

Finally, the paper by C. Colapinto and E. Benecchi uses a dynamic diffusion model of a TV series to analyze the evolution of electronic media transmissions and propose an optimal control model to assist the decision-maker with an optimal solution that balances the investment in a program's popularity sustenance and profit maximization.

\author{
Davide La Torre \\ Herb Kunze \\ Manuel Ruiz-Galan \\ Tufail Malik \\ Simone Marsiglio
}

\title{
References
}

[1] S. Lenhart and J. T. Workman, Optimal Control Applied to Biological Models, Mathematical and Computational Biology, Chapman \& Hall, Boca Raton, Fla, USA ;CRC Press, London, UK, 2007.

[2] M. Athans and P. L. Falb, Optimal Control: An Introduction to the Theory and Its Applications, Dover Publications, 2006.

[3] D. Liberzon, Calculus of Variations and Optimal Control Theory. A Concise Introduction, Princeton University Press, Princeton, NJ, USA, 2012.

[4] R. Vinter, Optimal Control, Birkhäuser, 2010.

[5] H. Schattler and U. Ledzewicz, Geometric Optimal Control: Theory, Methods and Examples, Springer, 2012.

[6] A. Chinchuluun, P. M. Pardalos, R. Enkhbat, and I. Tseveendori, Eds., Optimization and Optimal Control: Theory and Applications, vol. 39, Springer, 2010.

[7] R. F. Stengel, Optimal Control and Estimation, Dover, New York, NY, USA, 1994.

[8] A. E. Bryson and Y. C. Ho, Applied Optimal Control, Hemispheres, 1975.

[9] P. Chen and S. M. N. Islam, Optimal Control Models in Finance: A New Computation Approach, Springer, 2005.

[10] L. Berkovitz and N. Medhin, Nonlinear Optimal Control Theory, Chapman \& Hall, CRC Press, 2012.

[11] S. Aniţa, V. Arnăutu, and V. Capasso, An Introduction to Optimal Control Problems in Life Sciences and Economics: From Mathematical Models to Numerical Simulation with MATLAB, Birkhäuser, 2011.

[12] H. P. Geering, Optimal Control with Engineering Applications, Springer, 2007. 


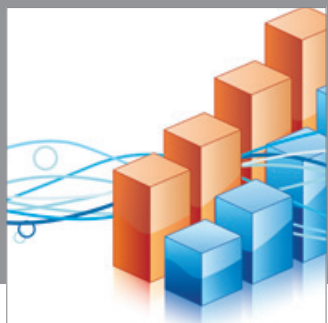

Advances in

Operations Research

mansans

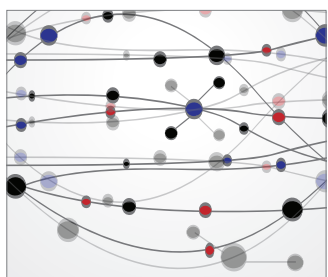

The Scientific World Journal
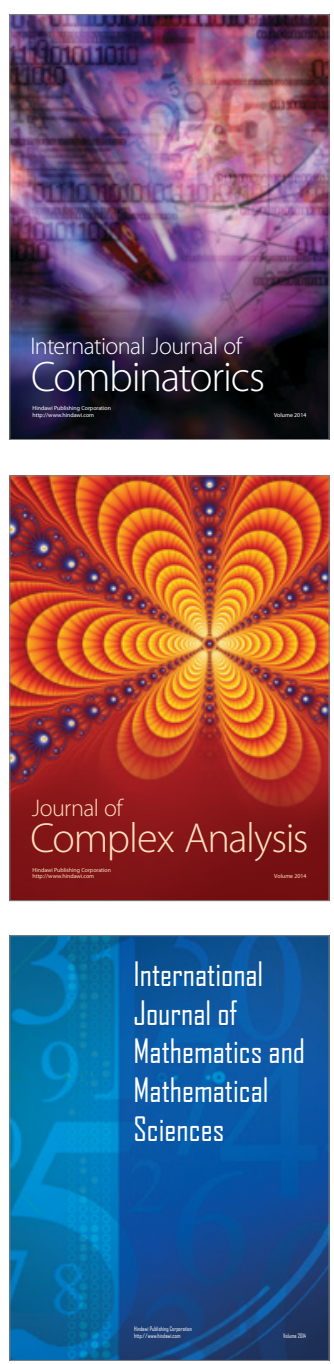
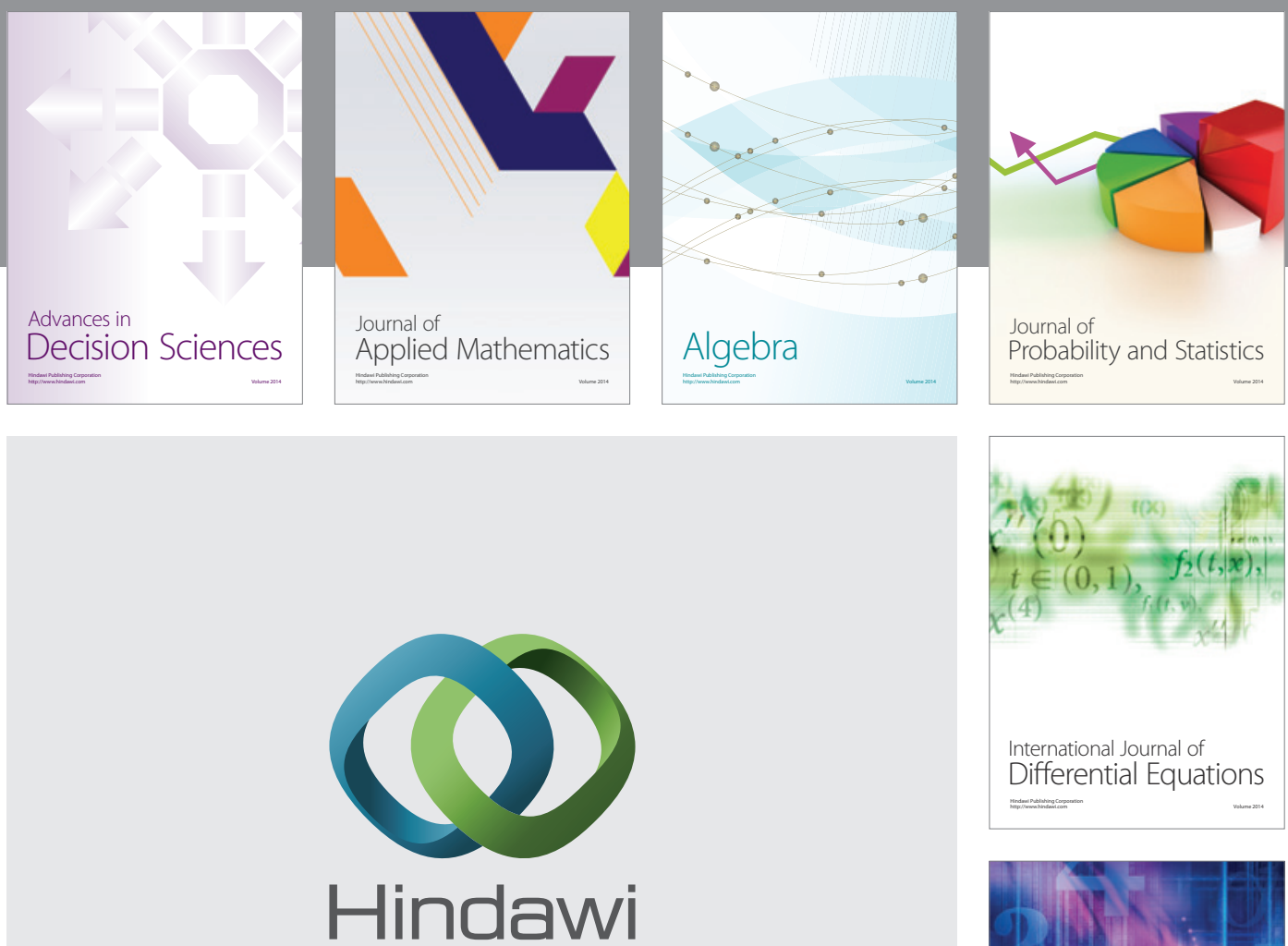

Submit your manuscripts at http://www.hindawi.com
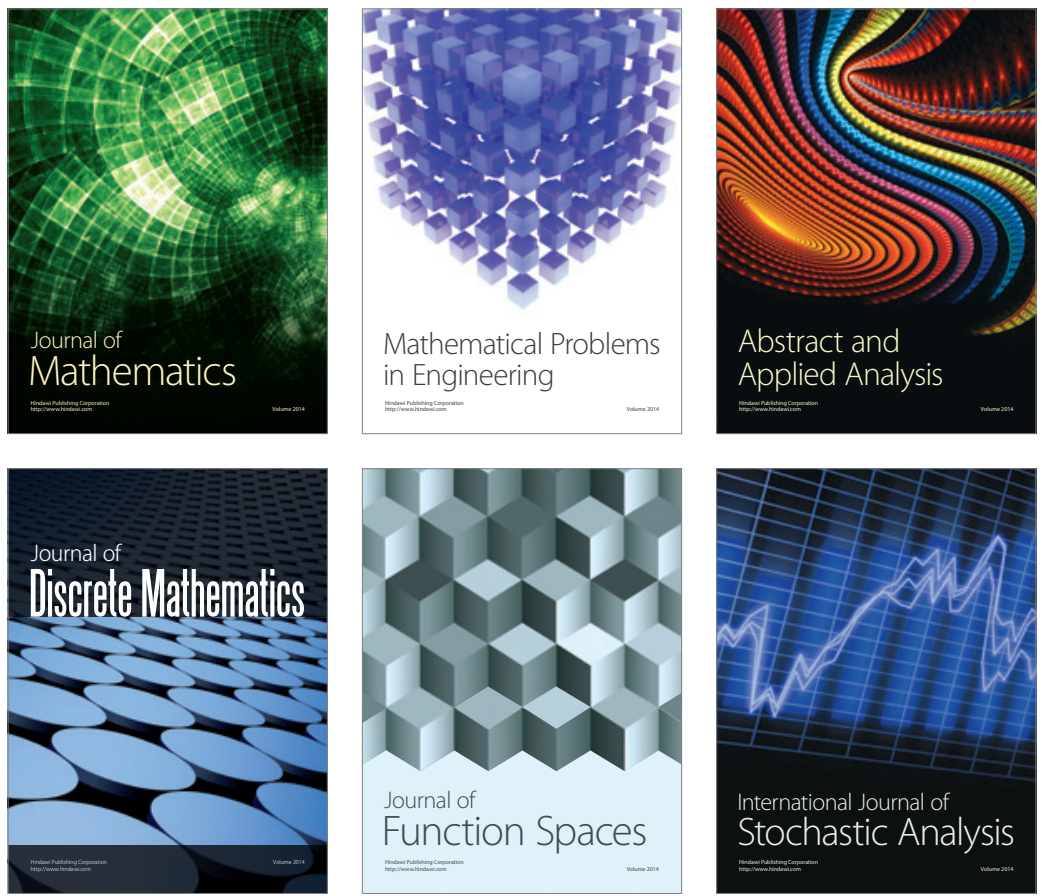

Journal of

Function Spaces

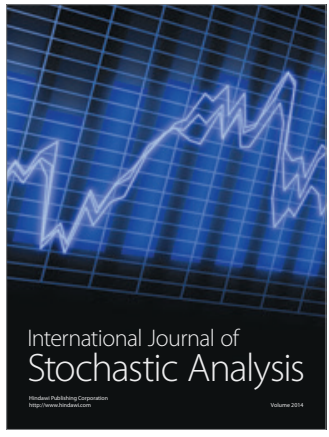

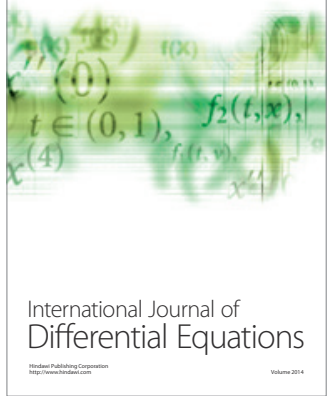
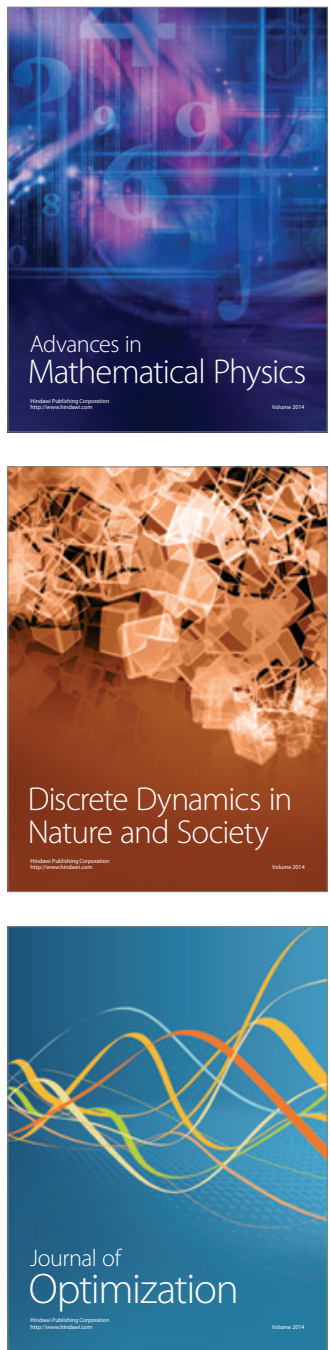